\title{
Significance of Frequency Analysis in X-ray Reflectivity: Towards analysis which does not depend too much on models
}

\author{
Kenji Sakurai, Mari Mizusawa and Masashi Ishii \\ National Institute for Materials Science, Sengen, Tsukuba, Ibaraki 305-0047 Japan \\ Fax: 81-29-859-2801, e-mail: sakurai@yuhgiri.nims.go.jp
}

\begin{abstract}
The present paper discusses the feasibility of frequency analysis of X-ray reflectivity data, which have been used extensively to obtain information on density, layer thickness, surface and interface roughness for layered materials. So far, the experimentally obtained data have been analyzed by least squares fitting to the theoretical reflectivity curve for assumed layered structures. As is often the case with non-linear systems, the solution is not generally unique. In fact, it is sometimes not easy to judge which structural parameters should be selected from several promising candidates that differ from each other to a fair extent but agree quite well with the experimental curve. The problem becomes even worse when one is not confident in the model, i.e., the number of layers etc. Assuming that several additional layers could improve the degree of fit, this raises the question of whether such a model should be accepted or not. The advantages of using frequency analysis, such as Fourier and Wavelet transform techniques, is that one can start from analysis which does not rely on the model too much. As the analysis gives a rough sketch of the electron density profile of the sample, one can decide which models should and should not be chosen. Furthermore, filtering specific frequency components of the data can help to see some morphology changes in the specific interface.

Key words: surface, interface, interference fringe, Fourier transform, Wavelet transform
\end{abstract}

\section{INTRODUCTION}

$\mathrm{X}$-ray reflectivity is a powerful method for the non-destructive analysis of the structure along the depth of thin films and layered materials [1-6]. As the interference fringes observed in the reflectivity curve relate in both sensitivity and quantity to the small changes in layer thickness, some consideration has even been given to standardizing the technique to determine layer thickness in industrial applications [7, 8]. Data analysis, so far, has been very frequently done by so-called whole-pattern fitting of the theoretical reflectivity based on Parratt's formalism [9] combined with Nevot-Croce roughness approximation [10]. The method basically works very well as long as the assumed model is good enough.

However, generally, the solution devised using non-linear least squares fitting is not unique. There are also a number of local minima. The physical meaning of the parameters obtained needs to be examined, because these are not always justified based purely on a good fit. As several authors have reported [11-13], the use of genetic algorithms could be a help in some cases. On the other hand, as is the case for some complicated systems including soft matter, it is sometimes not easy to have a reliable model for fitting. Even the number of layers could be unknown or uncertain. Sometimes interfaces are graded, and thus unsuitable for description under Nevot-Croce's Gaussian-type roughness. One should be careful, because some good fits are possible even in such cases.

The present paper intends to demonstrate that the use of frequency analysis, such as Fourier and Wavelet transform techniques, could sometimes help in carrying out analysis which does not rely on the model too much. The analysis gives a rough sketch of the electron density profile of the sample. One can use this to prepare a reasonable model to attempt fitting to the experimentally obtained reflectivity curve. Otherwise it is also possible to make direct use of such a profile for some inversion approach based on phase guessing $[14,15]$. Moreover, as each frequency component of the interference fringes corresponds to each pair of interfaces, frequency filtering would be useful for observing specific interfaces in multilayer cases. The combined use of the above frequency analysis and conventional fitting procedures would make X-ray reflectivity data more feasible for realistic analysis.

\section{MANUAL FREQUENCY ANALYSIS}

It was early in the $20^{\text {th }}$ century that determination of layer thickness was first attempted by X-ray reflectivity. Figure 1 shows the $X$-ray reflectivity curve of a $142 \mathrm{~nm}$ thick Ni layer on glass, reported by Kiessig in $1931[16,17]$. The data were obtained by photographic recording, and then converted to X-ray intensity. At that time, direct reading of the frequency was the only possible analysis. The thickness was determined by simply reading the angle positions of each interference fringe maxima (or minima). The angle of the $m$ th ( $m$ is integer) maxima, $\theta_{\text {nax }}(m)$, and minima $\theta_{\min }(m)$, can be given as follows:

$\theta_{\max }(m)=\sqrt{\left(\frac{\left(m+\frac{1}{2}\right) \lambda}{2 d}\right)^{2}+\theta_{C}{ }^{2}}$ 


$$
\theta_{\min }(m)=\sqrt{\left(\frac{m \lambda}{2 d}\right)^{2}+\theta_{c}^{2}}
$$

where $\mathrm{d}, \lambda$ and $\theta_{\mathrm{c}}$ are layer thickness and $X$-ray wavelength, critical angle, respectively. Here, when the density of the layer is lower than that of the substrate, the formulas for $\theta_{\max }(m)$ and $\theta_{\min }(m)$ are changed for each other. One can plot $\theta_{\max }(m)^{2}$ as a function of $m^{2}$ (or $\left.(m+1 / 2)^{2}\right)$, and then the slope and the intercept of the straight line give $(\lambda / 2 \mathrm{~d})^{2}$ and $\theta_{\mathrm{c}}{ }^{2}$, respectively. As the relationship is simple and feasible, the method can be used even in modern industrial applications. When only the layer thickness is the main interest, this method works well, because it does not need to assume any models for whole pattern fitting. It is interesting that several papers on the applications of manual frequency analysis were published $[18,19]$ just after the completion of Parratt's theory of X-ray reflectivity from multilayers.

\section{FOURIER TRANSFORM}

\subsection{Fundamental issues}

The Fourier transform technique [20-29] is an extension of the manual frequency analysis described in the previous section. According to Parratt's formalism [9], X-ray reflectivity can be calculated by using a recursive equation. The reflection coefficient for the interface between $(n-1)$ th and $n$th layers, $R_{n-1, n}$ can be given using that for deeper interface, $\mathrm{R}_{\mathrm{n}, \mathrm{n}+1}$ as follows:

$$
R_{n-1, n}=a_{n-1} 4\left(\frac{R_{n, n+1}+F_{n-1, n}}{R_{n, n+1} F_{n-1, n}+1}\right)
$$

where $a_{n-1}$ and $F_{n-1, n}$ are the amplitude factor for the (n-1) th layer and the Fresnel coefficient for the (n-1, n) interface, respectively. Let's look at the simplest case - single film on the substrate, where the medium numbers 1,2, and 3 are vacuum (air), film, and the substrate, respectively. Here, the film thickness is $\mathrm{d}_{2}$. The X-ray reflectivity, which is a function of scattering vector $\mathrm{q}_{z}(=4 \pi \theta / \lambda)$, becomes the following:

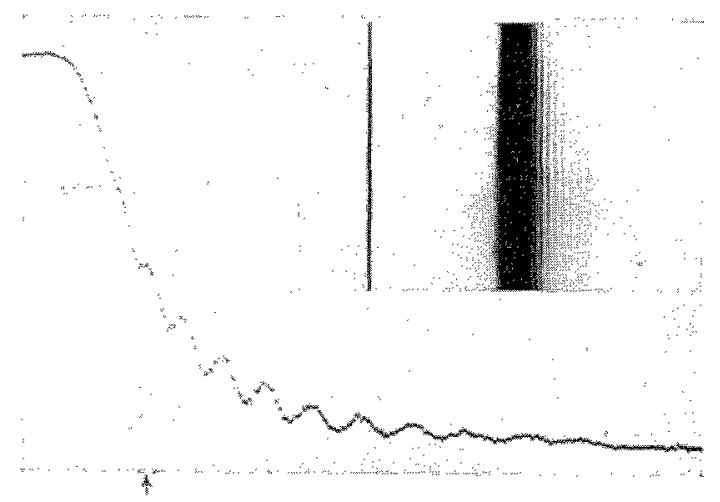

Fig. $1 \mathrm{X}$-ray reflectivity curve reported in the early days of X-ray physics. The sample is Ni $142 \mathrm{~nm}$ thin film prepared on glass substrate, and $\mathrm{Ni} \mathrm{K \alpha _{1 }} \mathrm{X}$-rays $(0.1655$ $\mathrm{nm}$ ) are used. (Reproduced with permission from [17], Fig.2 (photo shown as inset) and Fig.3 (curve))

$$
\begin{aligned}
R\left(q_{z}\right) & =\left|R_{1,2}\right|^{2} \\
& =\left|\frac{R_{2,3}+F_{1,2}}{R_{2,3} F_{1,2}+1}\right|^{2} \\
& =\left|\frac{\operatorname{Exp}\left(-i \gamma_{2}\right) F_{2,3}+F_{1,2}}{\operatorname{Exp}\left(-i \gamma_{2}\right) F_{2,3} F_{1,2}+1}\right|^{2}
\end{aligned}
$$

where

$$
\begin{aligned}
\gamma_{2} & =\sqrt{q_{z}^{2}-q_{c}^{2}} d_{2} \\
& =q_{z}^{\prime} d_{2}
\end{aligned}
$$

Here $q_{c}$ is the critical scattering vector. Though the exact expression of $\gamma_{2}$ includes the absorption term, this becomes negligible, when $R\left(q_{z}\right)$ is clearly smaller than 1. Therefore, in the $q_{z}$ region where interference fringes are observed, the Eq. (4) can be rewritten as follows:

$$
R\left(q_{z}^{\prime}\right)=\frac{F_{1,2}^{2}+F_{2,3}^{2}+2 F_{1,2} F_{2,3} \cos \left(q_{z}^{\prime} d_{2}\right)}{\left(1-F_{1,2}^{2}\right)\left(1-F_{2,3}^{2}\right)}
$$

As the oscillating part is a simple cosine function of $\mathrm{q}_{z}{ }^{\prime} \mathrm{d}_{2}$, one can obtain a single peak corresponding to $\mathrm{d}_{2}$ by its Fourier transform [20-22]. For the sake of simplicity, surface and interface roughness are not included in the equation. However, roughness does
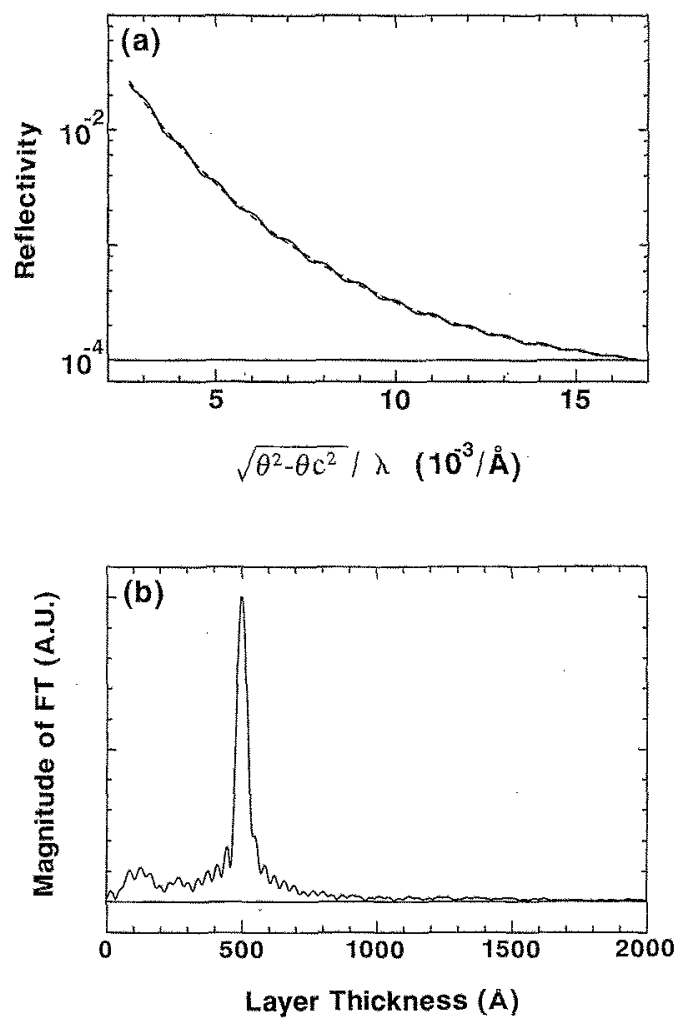

Fig.2 Thickness determination of $\mathrm{SiO}_{2}$ layer $(50.1 \mathrm{~nm})$ formed on Si substrate by Fourier transform of X-ray reflectivity with $8 \mathrm{keV}$ synchrotron photons. (a) X-ray reflectivity plotted as a function of $\mathrm{q}_{z}^{\prime}$ considering critical angle correction. (b) Magnitude of Fourier transform of the oscillating part extracted from the curve (a). (Reproduced with permission from [21], Fig.2) 
not affect the periodicity of the fringes. The influence is restricted to dampening the amplitude of the interference fringes and modifying the whole decay rate. Figure 2 shows an example for the case of $\mathrm{SiO}_{2} / \mathrm{Si}$. First, one should plot the data as a function of $\mathrm{q}_{\mathrm{z}}^{\prime}$, and then estimate an average curve (shown as a dashed line) in logarithmic scale. Then, the oscillation can be extracted by normalization. The magnitude of Fourier transform has a sharp symmetrical peak. The position agrees with the layer thickness.

If a wrong critical angle is given, what happens? Here, errors in $\mathrm{q}_{\mathrm{z}}{ }^{\prime}$ and $\mathrm{q}_{\mathrm{c}}$ are $\Delta \mathrm{q}_{\mathrm{z}}{ }^{\prime}$ and $\Delta \mathrm{q}_{\mathrm{c}}$, respectively. Then the contents of the cosine part become as follows:

$$
\begin{aligned}
\left(q_{z}^{\prime}+\Delta q_{z}^{\prime}\right) d_{z} & =q_{z}^{\prime}\left(d_{2}+\frac{\Delta q_{z}^{\prime}}{q_{z}^{\prime}} d_{2}\right) \\
& =q_{z}^{\prime}\left(d_{2}-\frac{q_{c} \Delta q_{c}}{q_{z}^{\prime 2}} d_{2}\right)
\end{aligned}
$$

This results in a shift of the peak position, and also makes the peak asymmetrical. In other words, only when $\Delta \mathrm{q}_{\mathrm{c}}=0$ one can obtain a perfectly symmetrical peak with no shift. It is possible to find this correct condition by changing $\mathrm{q}_{\mathrm{c}}$ with a step of around $0.5 \sim 1$ $\times 10^{-3} \mathrm{~nm}^{-1}$, which corresponds to ca. 0.1 mrad step for $8 \mathrm{keV} \mathrm{X}$-rays.

The above frequency analysis by Fourier transform is even more useful in cases where there are rather many layers. X-ray reflectivity for double films on the substrate can be written as follows [22]:

$$
\begin{aligned}
R\left(q_{z}\right)= & \left(A \cos \gamma_{2}+B \cos \gamma_{3}+C \cos \left(\gamma_{2}+\gamma_{3}\right)\right. \\
& \left.+D \cos \left(\gamma_{2}-\gamma_{3}\right)+E\right) / F
\end{aligned}
$$

where

$$
\begin{aligned}
& A=2 F_{1,2} F_{2,3}\left(1+F_{3,4}^{2}\right), \quad B=2 F_{2,3} F_{3,4}\left(1+F_{1,2}^{2}\right), \\
& C=2 F_{1,2} F_{3,4}, \quad D=2 F_{1,2} F_{2,3}^{2} F_{3,4}, \\
& E=F_{1,2}^{2}+F_{2,3}^{2}+F_{3,4}^{2}+F_{1,2}^{2} F_{2,3}^{2} F_{3,4}^{2}, \\
& F=\left(1-F_{1,2}^{2}\right)\left(1-F_{2,3}^{2}\right)\left(1-F_{3,4}^{2}\right)
\end{aligned}
$$

In this case, the factor D is negligible, and therefore, 3 frequency components will appear in the interference fringes of the reflectivity curve. They basically correspond to each layer thickness and the sum. To determine $d_{2}$ and $d_{3}$, the oscillation in $R\left(q_{z, 2}\right)$ and $R\left(q_{z, 3^{1}}\right)$, respectively, should be Fourier transformed. Here $\mathrm{q}_{z, n}{ }^{\prime}(\mathrm{n}=2$ or 3 ) is given as follows:

$$
q_{z, n}^{\prime}=\sqrt{q_{z}^{2}-q_{c, n}^{2}}
$$

When the layer number becomes $\mathrm{N}-1$, the number of interfaces is $\mathrm{N}$. The number of frequency components included in the interference fringes is ${ }_{\mathrm{N}} \mathrm{C}_{2}=\mathrm{N}(\mathrm{N}-1) / 2$. Therefore, basically it is possible to know $\mathrm{N}$ even for completely unknown samples, although there appear to be limitations where several layers are of the same or very similar thickness.

Figure 3 shows the calculation in the case of $\mathrm{Cu}[50 \mathrm{~nm}] / \mathrm{Al}[23 \mathrm{~nm}] / \mathrm{Au}[12 \mathrm{~nm}] / \mathrm{Si}$. Although Fourier transform for $R\left(\mathrm{q}_{\mathrm{z}, 2}\right)$ gives a correct value only for $d_{2}$, i.e., $\mathrm{Cu}$ layer thickness, other peaks for
$A 1$ and $A u$ are not so bad. Besides Fourier transform for $R\left(q_{z, 3^{\prime}}\right)$ and $R\left(q_{z, 4^{\prime}}\right)$, one could just try whole pattern fitting by using such layer thickness as initial values. As shown in the inset of Fig. 3, in this case, the first derivative of the electron density gives the position of the interface. In the kinematical approach, as the derivative of electron density profile is related to Fourier transform [3,5], inverse Fourier transform of the power spectrum, i.e, autocorrelation function can be discussed similarly. Some good instructive accounts on the relationship between the present frequency analysis and the electron density profile are found elsewhere [24].

For the above frequency analysis, one can employ the ordinary Cooley-Tukey FFT algorithm [30]. Let us assume that the extracted oscillating part has the data-point interval of $\Delta q_{\mathrm{z}}^{\prime}$ and the FFT's spectrum size is $M$. Then in Fourier space, the data-point interval $\Delta d$ and the spectrum size $d_{\text {max }}$ are given as follows:

$$
\Delta d=\frac{1}{2 M \Delta q_{z}^{\prime}}, \quad d_{\max }=\Delta d\left(\frac{M}{2}-1\right)
$$
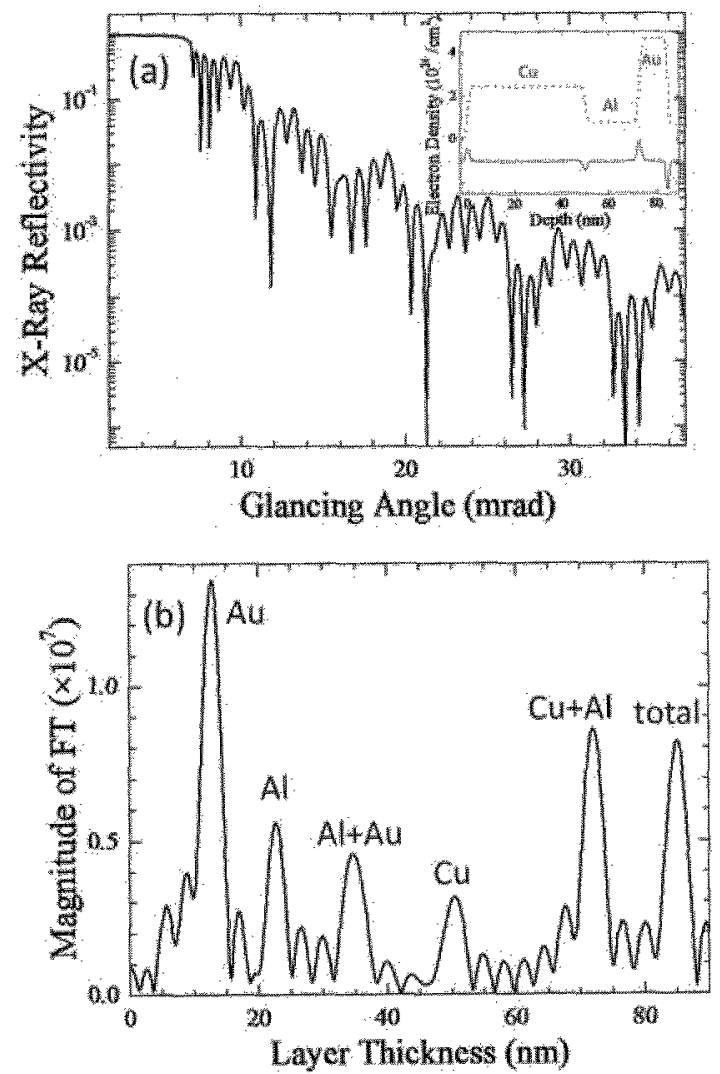

Fig.3 Simulation of X-ray reflectivity curve for $\mathrm{Cu}[50 \mathrm{~nm}] / \mathrm{A}[23 \mathrm{~nm}] / \mathrm{Au}[12 \mathrm{~nm}] / \mathrm{Si}$ case. (a) X-ray reflectivity, (b) Magnitude of Fourier transform, after $\mathrm{q}_{\mathrm{z}}{ }^{\prime}$ correction with $\theta_{\mathrm{c}}=6.8 \mathrm{mrad}$ (for $\mathrm{Cu}$ ). The rms roughness for the surface, the $\mathrm{Cu} / \mathrm{Al}, \mathrm{Al} / \mathrm{Au}$, and $\mathrm{Au} / \mathrm{Si}$ interfaces are assumed as $0.5,0.5,0.5$ and $0.35 \mathrm{~nm}$, respectively. In the inset of (a), the electron density profile (dashed line) and the derivative (solid line) are drawn. Here, the number of interfaces is 4 , and one can see 6 peaks in Fourier transformed data. The peak for $\mathrm{Cu}$ gives correct thickness, $50 \mathrm{~nm}$. 
When $M$ is 14 bit $\left(2^{14}=16384\right)$, the typical values would be $\Delta \mathrm{d}=0.1 \sim 0.2 \mathrm{~nm}$ and $\mathrm{d}_{\max }=0.8 \sim 1.6 \mu \mathrm{m}$, respectively. To give the layer thickness with high resolution to the order of $0.01 \mathrm{~nm}, \mathrm{M}$ needs to be larger. On the other hand, the peak position in Fourier space can be determined by direct integral calculation, instead of FFT algorithm [20].

\subsection{Applications and further developments}

Frequency analysis based on Fourier transform was first reported in 1991 [20-22]. Subsequently, quite a few research groups rediscovered the method independently, and reached the same conclusion [23-29]. Recently, some interesting extensions using anomalous dispersion were reported [31]. As the method is already mature, further applications will be of great importance [32-34].

Figures 4 and 5 show the effect of heating on a $\mathrm{Fe}[20 \mathrm{~nm}] / \mathrm{Si}$ sample, studied by X-ray reflectivity. One can see clear changes in the frequency of the interference fringe. As indicated in Fourier space, layer thickness changes by interdiffusion at the interface. Though the interface was at $20 \mathrm{~nm}$ originally, the position moved to $40 \mathrm{~nm}$. One can thus understand how powerful this type of frequency analysis is. In addition to layer thickness, it is possible to obtain further information on the structure, as an electron density profile, or the list of roughness values. Prior knowledge of the number of layers and total thickness is very helpful in obtaining a satisfactory model with some good initial parameters for whole pattern fitting. As for the interdiffusion observed in (b) in Figs. 4 and 5, the authors also employed secondary ion mass spectroscopy (SIMS) to confirm the results. Perhaps another promising alternative would be the measurement of $\mathrm{Fe} \mathrm{Ka}$ fluorescent $\mathrm{X}$-rays as a function of the glancing angle $[1,35,36]$, during the measurement of X-ray reflectivity shown in Fig. 4.

\section{WAVELET TRANSFORM}

\subsection{Beyond Fourier transform}

As described earlier, frequency analysis using Fourier transform is simple, quick and feasible. However, there appear to be some problems. Fourier transform is usually done with a single wide window, i.e., for almost all the measured q-range. This means that the results just tell us the list of layer thicknesses in the sample, but generally do not give the layering order. For example, in the case of Fig.3 (b), it is not always clear which layer, $\mathrm{Au}$ or $\mathrm{Al}$, is the $2^{\text {nd }}$ layer (although in this case one could estimate the order to some extent by evaluating the magnitude of the peak). Other possible concerns are further analysis other than just determination of the layer thickness. As such concerns mainly reflect the very wide single window for Fourier analysis, one might think about analysis using narrowly divided sliding windows. This is known as short segment (or time) Fourier transform (SSFT or STFT) in the field of electronic signal processing [37]. However, the SSFT of X-ray reflectivity would suffer from very low quality spectra with substantial noise.

Therefore, one promising solution is Wavelet transform $[38,39]$. The method uses sliding spectral windows in the same way as SSFT, but each width can be changed automatically depending on the amplitude. This helps to keep the signal-to-noise ratio to an acceptable level. The Wavelet transform of the X-ray reflectivity data $R\left(q_{z}{ }^{\prime}\right)$ can be defined as follows:
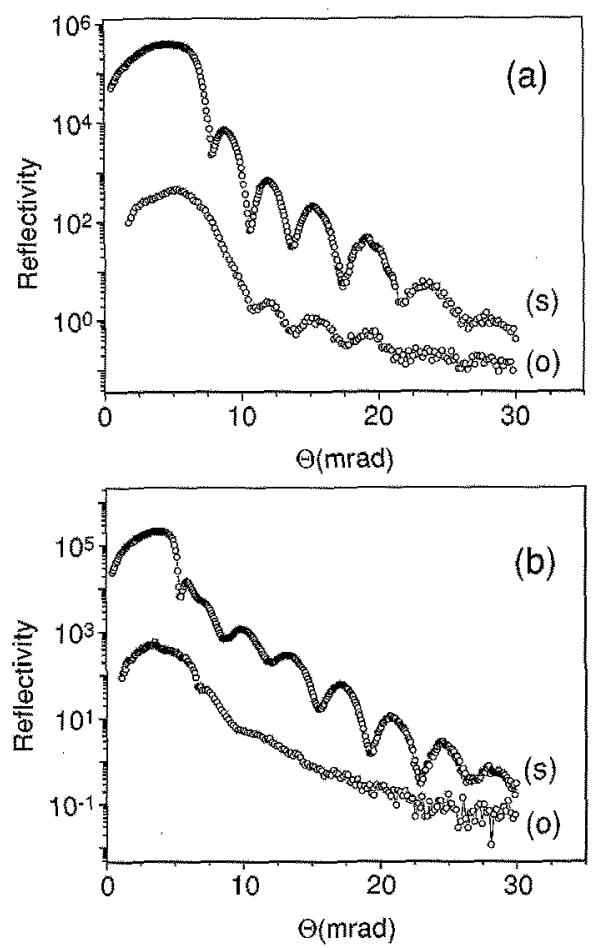

Fig.4 X-ray specular (s) and off-specular (o) reflectivity measured for iron thin film deposited on silicon substrate; (a) before heating, (b) after heating. (Reproduced with permission from [32], Fig.1)

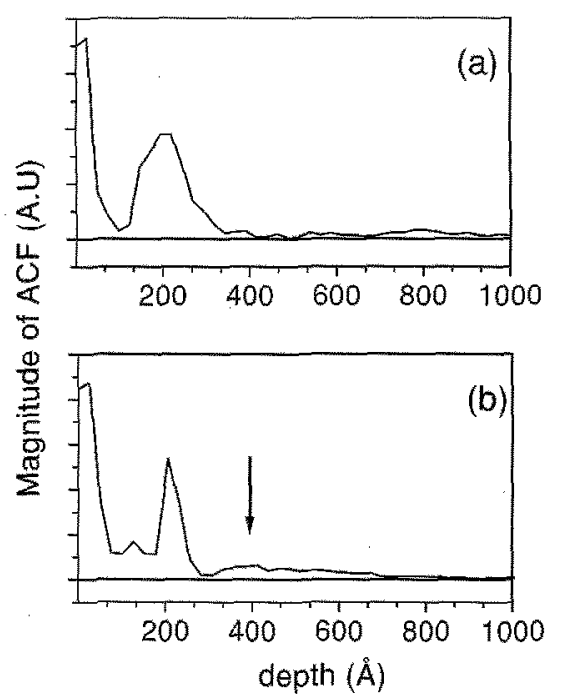

Fig.5 Frequency analysis of X-ray reflectivity data shown in Fig. 4; before (a) and after (b) heating. The total thickness before heating was $20 \mathrm{~nm}$ as shown in (a). The broad peak in (b) indicated by arrow suggests that there is interdiffusion at the $\mathrm{Fe} / \mathrm{Si}$ interface, leading to the increase of effective film thickness to 40 nm. (Reproduced with permission from [32], Fig.2) 
$W T(a, b)=\frac{1}{\sqrt{a}} \int R\left(q_{z}^{\prime}\right) g\left(\frac{q_{z}^{\prime}-b}{a}\right) d q_{z}^{\prime}$

where $a$ and $b$ are the scaling and position parameters, respectively, of the mother wavelet function. $g$. Note that Eq. (12) essentially represents the autocorrelation function, exhibiting peak at $\dot{\mathrm{q}}_{\mathrm{z}}^{\prime}=b$, when the parameter $a$ is fixed. So far, various mother wavelet functions have been developed, with one of the simplest being the Mexican Hat wavelet, which can be written as follows:

$g(t)=\left(1-t^{2}\right) \exp \left(-\frac{t^{2}}{2}\right)$

As is clear from the function form, it is suitable for simple cosine type oscillations observed in X-ray reflectivity.

Advanced frequency analysis of X-ray reflectivity data, based on the above Wavelet transform, was first reported in 2000, by Smigel and Conet [38]. Figures 6 and 7 show the analysis of X-ray reflectivity data for oxidized iron thin film during heating. In this case, the interference fringes include 3 frequency components, which indicate 2 layers on the substrate. As discussed earlier, Fourier transform can show this clearly with ease, but does not give the order, either which comes top or $2^{\text {nd }}$. On the other hand, with Wavelet transform, one can do frequency analysis during $q_{z}^{\prime}$ scan, leading to quantitative knowledge on $\mathrm{q}_{\mathrm{z}}{ }^{\prime}$ or grazing angle, and layer thickness. As illustrated in Fig. 7, a 3D picture of the autocorrelation function demonstrates that the top and the $2^{\text {nd }}$ layers are $8 \mathrm{~mm}$ and $30.7 \mathrm{~nm}$, respectively, because the latter appears at a higher grazing angle than around $0.25 \mathrm{deg}$.

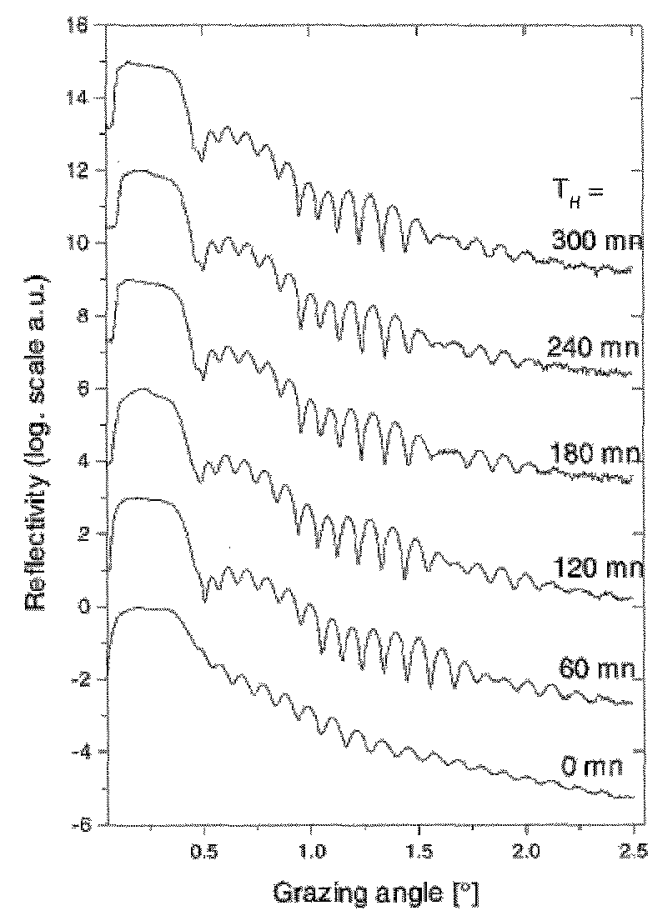

Fig.6 X-ray specular reflectivity of oxidized iron thin film on synthetic quartz substrate with different heating time $\left(\mathrm{T}_{\mathrm{H}}\right)$. (Reproduced with permission from [38], Fig.7)

\subsection{Frequency filtering}

As the $X$-ray reflectivity curve is a complicated mixture of information on the surface and all interfaces, generally, analysis only for the specific interface is not easy. There has been an apparent limitation in the case of the whole pattern fitting approach. Therefore, the use of Wavelet transform's capability of frequency filtering is a very interesting direction [39-41]. It gives detailed analysis of the specific interface of the multilayer case.

To analyze the specific interface by Wavelet transform, first one needs to choose the layer, by fixing $b$ parameter as some peak position. Then the Wavelet transform becomes WT $(a)$, a simple function of the scale parameter, $a$. On the other hand, layer selection corresponds to specifying a pair of interfaces, above or below the layer. Here, if we call each roughness $\sigma_{1}$ and $\sigma_{2}$, respectively, then one can define a new parameter, $\sigma_{12}=\left(\sigma_{1}^{2}+\sigma_{2}^{2}\right)^{1 / 2}$, which may be termed 'layer roughness'. When some kinematical approach is accepted, WT $(a)$ is simplified, and is expressed by only 2 variables including $\sigma_{12}$. Then the curve fitting can be done for experimental Wavelet transformed data. Accordingly $\sigma_{12}$ can be determined without assumptions on the other structure parameters of the layered materials. The method is feasible, but substantially in need of further development.

\section{CONCLUSIONS AND FINAL REMARKS}

In summary, frequency analysis of X-ray reflectivity data is feasible and significant, particularly when the main interest is focused on

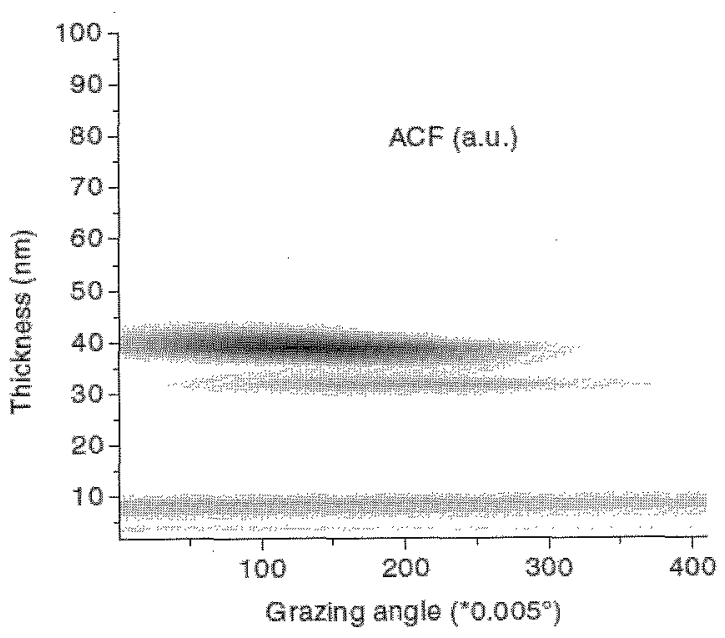

Fig.7 Wavelet transform of X-ray reflectivity for the sample with 300 min heating time shown in Fig.6. The autocorrelation function (ACF) of the derivative of electron density profile is given in $3 \mathrm{D}$ picture, as a function of both thickness and grazing angle, i.e., $\mathrm{q}_{z}{ }^{\prime}$. As the frequency components found in interference fringes correspond to the layer thickness, this shows when (at which angle) each frequency component appears. One can see that $8 \mathrm{~nm}$ is visible all the time, but $30.7 \mathrm{~nm}$ appears later at a higher angle than around $0.25 \mathrm{deg}$ (Reproduced with permission from [38], Fig.9) 
specific layers and/or interfaces, and when some careful analysis is necessary without depending too much on models that assume many uncertain parameters. Historically, manual frequency analysis has played an important role since Kiessig's discovery of the interference fringe, and is still useful even in the $21^{\text {st }}$ century, for the analysis of the single layer case. Modern frequency analysis uses Fourier and Wavelet transforms. This can give the sketch of a whole layered structure quickly without assuming any models, and the frequency filtering capability helps when looking at a specific interface. On the other hand, conventional least squares fitting is indispensable in many cases to determine all structure parameters for the given model. Their combined use will make $\mathrm{X}$-ray reflectivity more reliable in realistic analysis.

\section{ACKNNOWLEDGMENTS}

The authors would like to thank Professor A. Iida for his continuous encouragement to the present research. Their thanks also go to Drs. W. Yashiro and Y. Yano for their helpful discussion on the feasibility of Fourier and Wavelet transforms in X-ray reflectivity.

\section{REFERENCES}

[1] D. K. G. de Boer, A. J. G. Leenaers, and W. W. van den Hoogenhof, X-ray Spectrom., 24, 91-102 (1995).

[2] J. Daillant and A. Gibaud Eds., "X-ray and Neutron Reflectivity: Principles and Applications", Springer (1999).

[3] M. Tolan, "X-ray Scattering from Soft-Matter Thin Films", Springer (1999).

[4] K. Stoev and K. Sakurai, Spectrochim. Acta, B54, 41-82 (1999).

[5] J. Als-Nielsen and D. McMorrow, "Elements of Modern X-ray Physics", John Wiley \& Sons (2000).

[6] U. Pietsch, V. Holy and T. Baumbach, "High-Resolution X-ray Scattering: From Thin Films to Lateral Nanostructures", Springer (2004).

[7] T. Fujimoto, B. Li and I. Kojima, Journal of NIMC, 7, 335-346 (1999). (in Japanese)

http://www.aist.go.jp/NIMC/publication/journal99/76-3.html

[8] P.Colombi, D.K.Agnihotri, V.E.Asadchikov, E.Bontempi, D.K.Bowen, C.H.Chang, L.E.Depero, M.Farnworth, T.Fujimoto, A.Gibaud, M.Jergel, M.Krumrey, T.A.Lafford, A.Lamperti, T.Ma, R.J.Matyi, M.Meduna, S.Milita, K.Sakurai, L.Shabel'nikov, A.Ulyanenkov, A. Van der Lee and C.Wiemer, J. Appl. Cryst., 41, 143-152 (2008).

[9] L. G. Parratt, Phys. Rev., 95, 359-369 (1954).

[10] P. Croce and L. Nevot, J. Appl. Cryst., 7, 125-130 (1974); L. Nevot and P. Croce, Rev. Phys. Appl., 15, 761-779 (1980).

[11] M. Wormington, C. Panaccione, K. M. Matney and K. Bowen, Phil. Trans. R. Soc. Lond., A357, 2827-2848 (1999).

[12] A. Ulyanenkov and S. Sobolewski, J. Phys. D, A38, 235-238 (2005).

[13] J. Tiilikainen, J-M. Tilli, V. Bosund, M. Mattila, T. Hakkarainen, V-M. Airaksinen and H. Lipsanen, J.
Phys. D, 40, 215-218 (2007).

[14] V.-O. de Haan, A. A. van Well, S. Adenwalla and G. P. Felcher, Phys. Rev., B52, 10831-10833 (1995)

[15] M. K. Sanyal, J. K. Basu, A. Datta, and S. Banerjee, Europhys. Lett., 36, 265-270 (1996).

[16] H. Keissig, Ann. Phys., 10, $715-768$ (1931).

[17] H. Keissig, Ann. Phys., 10, 769-788 (1931).

[18] N. Wainfan, N. J. Scott and L. G. Parrat, J. Appl. Phys., 30, 1604-1609 (1959).

[19] J. P. Sauro, J. Bindell and N. Wainfan, Phys. Rev., 143, 439-443 (1966).

[20] K. Sakurai and A. Iida, The Pacific International Congress on X-ray Analytical Methods (August 15-19, 1991).

[21] K. Sakurai and A. Iida, Jpn. J. Appl. Phys., 31, L113-115 (1992)

[22] K. Sakurai and A. Iida, Adv. in X-Ray Anal., 35, 813-818 (1992).

[23] F. Bridou and B. Pardo, J. Phys. III (France), 4, 1523 (1994); J. X-Ray Sci. Technol., 4, 200-216 (1994); J. Phys. III (France), 6, C4 367-383 (1996).

[24] F. Bridou, J. Gautier, F. Delmotte, M.-F. Ravet, O. Durand and M. Modreanu, Appl. Surf. Sci, 253, 12-16 (2006).

[25] G. Vignaud, A. Gibaud, G. Grubel, S. Joly, D. Ausserre, J. F. Legrand, and Y. Gallot, Physica, B248, 250-257 (1998).

[26] D. Rafaja, v. Valvoda, T. Sikola, and J. Spousta, Thin Solid Films, 324, 198-208 (1998).

[27] M. A. Marcus, Appl. Phys. Lett., 72, 659-661 (1998).

[28] O. H. Seeck, I. D. Kaendler, M. Tolan, K. Shin, M. H. Rafailovich, J. Sokolov, and R. Kolb, Appl. Phys. Lett., 76, 2713-2715 (2000).

[29] L. G. de Peralta and H. Temkin, J. Appl. Phys., 93, 1974-1977 (2003).

[30] J. W. Cooley and J. W. Tukey, Math. of Comput., 19, 297-301 (1965).

[31] K. Ueda, Trans. MRS Japan, 32, 223-225 (2007).

[32] S. Banerjee, G. Raghavan, and M. K. Sanyal, J. Appl. Phys, 85, 7135-7139 (1999).

[33] C. E. Bouldin, W. E. Wallace, G. W. Lynn, S. C. Roth, and W. L. Wu, J. Appl. Phys., 88, 691-695 (2000).

[34] H. Takeuchi, Y. Yamamoto, Y. Kamo, T. Kunii, T. Oku, T. Shirahama, H. Tanaka and $M$. Nakayama, J. Appl. Phys., 102, 043510 (2007).

[35] A. Iida, K.Sakurai and Y.Gohshi, Nucl. Instrum. \& Methods, A246, 736-738 (1986).

[36] K.Sakurai and A.Iida, Adv. in X-Ray Anal. 39, 695-700 (1997).

[37] M. Hino, "Spectral Analysis", Asakura, (1977) (in Japanese).

[38] E. Smigiel and A. Cornet, J. Phys. D, 33, 1757-1763 (2000).

[39] I. R. Prudnikov, R. J. Matyl, and R. D. Deslattes, J. Appl. Phys, 90, 3338-3346 (2001).

[40] O.Starykov and K.Sakurai, Appl. Surf. Sci., 244, 235-239 (2005).

[41] O. Durand and N. Morizet, Appl. Surf. Sci., 253, 133-137 (2006).

(Recieved December 10, 2007 ; Accepted June 30, 2008) 